\title{
Cytogenetic analysis of 130 renal oncocytomas identify three distinct and mutually exclusive diagnostic classes of chromosome aberrations
}

\author{
Christopher B. Anderson $^{1}$ | Michael Lipsky ${ }^{1}$ | Subhadra V. Nandula ${ }^{2}$ | \\ Christopher E. Freeman $^{3}$ | Thomas Matthews ${ }^{3}$ | Caitlin E. Walsh ${ }^{3}$ | Gen $\mathrm{Li}^{4}$ | \\ Matthias Szabolcs $^{3}$ | Mahesh M. Mansukhani ${ }^{3}$ | James M. McKiernan ${ }^{1}$ | \\ Vundavalli V. Murty ${ }^{3}$ (i)
}

\footnotetext{
${ }^{1}$ Department of Urology, Columbia University Irving Medical Center, New York, New York

${ }^{2}$ Cancer Genetics, Inc., Rutherford, New Jersey

${ }^{3}$ Department of Pathology and Cell Biology, Columbia University Irving Medical Center,

New York, New York

${ }^{4}$ Department of Biostatistics, Columbia University Irving Medical Center, New York, New York

\section{Correspondence}

Vundavalli V. Murty, Department of Pathology and Cell Biology, Columbia University Irving Medical Center, CHC 409, 3959 Broadway, New York, NY 10032

Email:vvm2@cumc.columbia.edu
}

\begin{abstract}
The cytogenetic alterations in renal oncocytoma $(\mathrm{RO})$ are poorly understood. We analyzed 130 consecutive RO for karyotypic alterations. Clonal chromosome abnormalities were identified in 63 (49\%) cases, which could be categorized into three classes of mutually exclusive cytogenetic categories. Class $1(\mathrm{~N}=20)$ RO had diploid karyotypes with characteristic 11q13 rearrangement in balanced translocations with 10 or more different chromosome partners in all cases. We identified recurrent translocation partners at $5 q 35,6 p 21,9 p 24,11 p 13-14$, and 11q23, and confirmed that CCND1 gene rearrangement at 11q13 utilizing fluorescence in situ hybridization (FISH). Class 2 RO ( $N=25$ ) exhibited hypodiploid karyotypes with loss of chromosome 1 and/or losses of $Y$ in males and $X$ in females in all cases. The class 3 tumors comprising of 18 cases showed diverse types of abnormalities with the involvement of two or more chromosomes exclusive of abnormalities seen in classes 1 and 2 tumors. Furthermore, karyotypically uninformative cases were subjected to FISH analysis to identify classes 1 and 2 abnormalities. In this group, we found similar frequencies of CCND1 rearrangement, loss of chromosome 1 or $\mathrm{Y}$ as with karyotypically abnormal cases. We validated our results against 91 tumors from the Mitelman database. Correlation of clinical data with all the three classes of ROs showed no clear evidence of overall patient survival. Our findings support the hypothesis that RO exhibit three principal cytogenetic categories, which may have different roles in initiation and/or progression. These cytogenetic markers provide a key tool in the diagnostic evaluation of RO.
\end{abstract}

\section{KEYWORDS}

$11 q 13$ rearrangement, cyclin D1, cytogenetics, loss of chromosome 1, renal oncocytoma

\section{1 | INTRODUCTION}

Renal oncocytoma (RO) accounts for $3 \%$ to $5 \%$ of all adult renal tumors. ${ }^{1} \mathrm{RO}$ with male predominance arises from intercalated cells of the collecting system and presents as both sporadic and familial forms. Oncocytomas are the most frequent renal tumors in patients with the Birt-Hogg-Dube syndrome caused by mutations in the folliculin gene. ${ }^{2}$ However, the oncogenic etiology of sporadic RO is not well understood. 
Clinically, RO are benign tumors typically occur as solitary lesions. Histological features of RO are usually distinctive but can overlap chromophobe renal cell tumors. ${ }^{3}$ Because chromophobe renal cell carcinoma (RCC) is a malignant tumor, differentiating RO from chromophobe is clinically important.

Only about 100 cases of RO with conventional karyotype have been reported in the literature (https://cgap.nci.nih.gov/Chromosomes/ CytList). ${ }^{4}$ Most of these studies were either single case reports or small case series, which identified loss of chromosomes $1, \mathrm{Y}$, and 14, and $11 q 13$ rearrangements as recurrent chromosome aberrations. However, the frequency of recurrent cytogenetic changes and their relation with each other has not been systematically examined.

We sought to identify recurrent chromosomal changes in RO by analyzing 63 karyotypically abnormal cases. We identified three mutually exclusive karyotype classes of ROs. Frequency of chromosome changes in the order of their occurrence was 11q13 rearrangement, loss of chromosome 1, loss of $Y$ in males and loss of $X$ in females, trisomy 7 , and loss of chromosomes 14,21 , and 22 . The present findings can be useful in differential diagnosis of RO and in understanding the role of genetic mechanisms in their pathogenesis.

\section{2 | MATERIALS AND METHODS}

\section{1 | Patient cohort}

A total of 130 consecutive RO specimens were subjected to karyotype analysis for diagnostic purposes at Columbia University Medical Center, New York, between 1999 and 2016. All patients had a radical or partial nephrectomy for a renal mass. We performed chart review to obtain clinical and histologic information on all the patients.

\section{2 | Karyotype and fluorescence in situ hybridization analyses}

We routinely perform karyotype and fluorescence in situ hybridization (FISH) analyses of all surgically removed renal masses at our institution Touch imprints were made and fixed immediately in methanol-acetic acid in majority of cases for FISH testing. Chromosome analysis was performed using standard methods. Briefly, fresh tumor specimens collected after surgery were washed with antimycotic solution $(0.5 \mu \mathrm{g} / \mathrm{mL}$ Amphotericin B) and antibiotics (200 IU/mL penicillin and $200 \mu \mathrm{g} / \mathrm{mL}$ streptomycin), minced with scalpel blade into fine pieces, and digested 2-12 hours with collagenase $(200 \mathrm{U} / \mathrm{mL})$ in complete Dulbecco's Modified Eagle's Medium (DMEM medium). Dissociated cells were washed twice and then cultured in complete DMEM medium supplemented with epidermal growth factor, insulin-transferrin-sodium selenate (Invitrogen). The cultures were monitored daily and appropriately confluent cells were subjected to metaphase preparations using standard methods after addition of colcemid for 12 hours. Metaphase preparations were subjected to Giemsa (GTG) banding and analyzed by standard methods. Karyotypes were described by the Standard Cytogenetic Nomenclature. FISH was performed either on touch imprints or cells processed for karyotype analysis using CCND1/CEP 11, CCND1 break apart, chromosome enumeration probes CEP X, CEP Y, CEP 1, and CEP 7 (Vysis, Downers Grove, IL) by standard methods. The cyclin D1 locus at 11q13 is labeled in SpectrumOrange fluorochrome in CCND1/CEP 11 cocktail spanning a $378-\mathrm{kb}$ physical distance equally covering on both $5^{\prime}$ and $3^{\prime}$ ends of the gene. When 11q13 rearrangement occurs, there is a spit in orange signal while SpectrumGreen labeled chromosome 11 serve as control to enumerate chromosome number. Dual color break apart CCND1 probe consists of two probes flanked by a centromeric $700-\mathrm{kb}$ probe labeled with SpectrumGreen and a telomeric 500-kb probe labeled with SpectrumOrange. This probe shows as orange-green signals together due to their close proximity in normal chromosome 11 and orange signal separated from green signal when 11q13 (CCND1) rearranged.

\section{3 | Data analysis}

Mitelman Database of Chromosome Aberrations and Gene Fusions in Cancer relates chromosome aberrations to tumor characteristics based on individual cases or associations. All ROs tumors reported until April 2016 in this database were retrieved by case quick searcher (http:// cgap.nci.nih.gov/Chromosomes/Mitelman). ${ }^{4}$ The database includes 91 karyotypically abnormal RO cases, excluding our previously published cases. ${ }^{5}$ Based on chromosomal abnormalities, we identified subgroups of patients. Pearson Chi-squared test and analysis of variance was performed to evaluate associations between clinical and demographic features, and different genetic characteristics.

\section{3 | RESULTS}

Of the 130 tumor specimens subjected to karyotype analysis, 63 (49\%) cases showed clonal chromosome abnormalities which include 11 previously reported cases. ${ }^{5}$ Normal karyotypes were found in $30(23 \%)$ cases and $37(29 \%)$ were failed to yield metaphases. The clinical, histologic, and cytogenetic features of karyotypically abnormal cases are shown in Table S1. Nearly all tumors (61 of 63) exhibited near-diploid range or pseudo-diploid karyotypes in their stem-line, while one had triploid karyotype and the other had 53 chromosomes. Ten tumors showed evidence for whole genome doubling (WGD). In general, RO exhibited simple karyotypes (less than four aberrations) with numerical loss of one or two chromosomes or $11 \mathrm{q} 13$ rearrangement. The most frequent chromosome aberration was the loss of a sex chromosome in 24 (38\%) cases ( $Y$ in $20 / 44,46 \%$ males; -X in $4 / 19,21 \%$ females). Other recurrent chromosomal abnormalities include loss or partial deletion of chromosome 1 (40\%), 11q13 rearrangement (32\%), trisomy 7 (18\%), monosomy 22 (11\%), monosomy 14 (10\%), and monosomy 21 (10\%) (Table 1).

\section{1 | Chromosome aberrations identify three mutually exclusive karyotypic classes of ROs}

The types of clonal chromosome abnormalities identified were allowed us to classify three karyotypically distinct classes of ROs (Table 2, Figure 1). Class 1 tumors showed $11 \mathrm{q} 13$ translocation involving 
TABLE 1 Recurrent chromosome alterations identified in oncocytoma in the present study and compared to Mitelman database

\begin{tabular}{lcrr}
\hline Abnormality & Present study $(\mathbf{N}=63)$ & Mitelman database (N = 91) & All (N = 154) \\
\hline Loss of $Y$ in males & $20 / 44(46 \%)$ & $26 / 52(50 \%)$ & $46 / 96(48 \%)$ \\
\hline Loss of $X$ in females & $4 / 19(21 \%)$ & $8 / 37(22 \%)$ & $12 / 56(21 \%)$ \\
\hline Loss of 1 or del 1p/1q & $25(40 \%)$ & $27(30 \%)$ & $52(34 \%)$ \\
\hline Trisomy 7 & $11(18 \%)$ & $13(15 \%)$ & $24(16 \%)$ \\
\hline Monosomy 14 & $6(9.5 \%)$ & $13(15 \%)$ & $19(12 \%)$ \\
\hline $11 q 13$ rearrangement & $20(32 \%)$ & $14(16 \%)$ & $34(22 \%)$ \\
\hline Monosomy 21q & $6(9.5 \%)$ & $8(9 \%)$ & $14(9 \%)$ \\
\hline Monosomy 22q & $7(11 \%)$ & $10(11 \%)$ & $17(11 \%)$ \\
\hline
\end{tabular}

TABLE 2 Comparison of types of chromosome abnormalities in various cytogenetic classes of renal oncocytoma in the present study and Mitelman database

\begin{tabular}{|c|c|c|}
\hline Abnormality & Present study & Mitelman database \\
\hline Class 1 & $N=20$ & $N=16$ \\
\hline $11 q 13$ rearrangement & $20 / 20$ (100\%) & $16 / 16(100 \%)$ \\
\hline Simple karyotype & $7 / 20$ (35\%) & 4/16 (31\%) \\
\hline Complex karyotype & $3 / 20(15 \%)$ & $3 / 16(19 \%)$ \\
\hline WGD & $2 / 20(10 \%)$ & 0 \\
\hline Class 2 & $N=25$ & $N=24$ \\
\hline Loss of 1 & 23 (92\%) & 23 (96\%) \\
\hline Del (1p) & 2 (8.0\%) & $1(4 \%)$ \\
\hline$-1,-Y$ & 12/17 (71\%) & $16 / 16(100 \%)$ \\
\hline$-1,-X$ & $3 / 8$ (38\%) & $5 / 8(63 \%)$ \\
\hline+7 & $6(24 \%)$ & $3 / 24(13 \%)$ \\
\hline-14 & $6(24 \%)$ & $10 / 24(42 \%)$ \\
\hline-21 & $5(20 \%)$ & $6 / 24(25 \%)$ \\
\hline-22 & $5(20 \%)$ & $5 / 24(21 \%)$ \\
\hline Simple karyotype & 22 (88\%) & 4/24 (17\%) \\
\hline Complex karyotype & $3(12 \%)$ & $5 / 24(21 \%)$ \\
\hline WGD & $8(32 \%)$ & $5 / 24(21 \%)$ \\
\hline Class 3 & $N=18$ & $N=51$ \\
\hline+7 & $5 / 18(28 \%)$ & 9/51 (18\%) \\
\hline$-Y$ or $-X$ & 7/18 (39\%) & 10/51 (20\%) \\
\hline Simple karyotype & $13(72 \%)$ & $32 / 51$ (63\%) \\
\hline Complex karyotype & 5 (28\%) & 10/51 (20\%) \\
\hline WGD & 0 & 0 \\
\hline
\end{tabular}

Abbreviation: WGD, whole genome doubling.

10 known and two unknown chromosome partners in all 20 tumors. Both recurrent (6p21 four cases, $5 q 35$ and 9p24 in three cases each, and 11q23 in two cases) and nonrecurrent (1p13, 4q27, 6q13, 7q11.2, $11 \mathrm{p} 14,15 \mathrm{q} 21$, and two unidentifiable chromosome regions) translocation partners were identified (Table 2, Figure 2). Seven (four with single abnormalities and three with two or more abnormalities) of the class 1 RO with 11q13 rearrangement also exhibited other chromosome abnormalities. These additional abnormalities were in the main line in four cases and three with loss of $Y$ in subclones. However, no recurrent copy number changes or structural chromosome rearrangements were seen among the additional abnormalities. Evidence for WGD was seen in 2 of $20(10 \%)$ tumors.

All 25 tumors in class 2 showed chromosome 1 abnormalities. Twenty-three tumors showed loss of chromosome 1, and two tumors showed deletion $1 \mathrm{p} 21$ and 1p22-p34, respectively. Among the other changes associated with monosomy 1 were loss of $Y$ chromosome in 12 of 17 (71\%) males and loss of $X$ chromosome in 3 of 8 (38\%) female patients. Other recurrent abnormalities in this group were trisomy 7 and monosomy 14 in six cases each, and loss of chromosomes 21 and 22 in five cases each (Table 2, Figure 1). Five cases showed additional isolated single chromosome abnormalities and three cases exhibited additional complex abnormalities in the form of chromosome gains or structural rearrangements associated with loss of chromosome 1. However, no evidence of recurrent structural rearrangements could be identified among these additional changes. Evidence for WGD was found in 8 of 25 (32\%) tumors.

Class 3 ROs consisted of 18 cases that had neither $11 q 13$ translocations nor chromosome 1 abnormalities. This group exhibited divergent types of chromosome abnormalities either as simple chromosome changes in 13 cases or complex chromosome abnormalities in 5 cases. The only recurrent numerical abnormalities were gain of chromosome 7 and loss of sex chromosomes in five cases each (Table 2, Figure 1). Among the structural abnormalities, $2 q 37$ rearrangements were seen in three cases and telomeric associations in three cases. No evidence of WGD was seen in this group.

\section{2 | FISH analysis of recurrent chromosome changes in classes 1 and 2 ROs}

We validated the karyotypic changes of loss of chromosomes $1, \mathrm{Y}, \mathrm{X}$, and 11q13 (CCND1) rearrangements by FISH. We tested 42 of 63 karyotypically abnormal cases by FISH based on the availability of materials. CCND1 rearrangement was found in all 15 class $1 \mathrm{RO}$ cases with $11 q 13$ translocation. None of the 10 class 2 or 3 RO lacking $11 q 13$ translocation were positive for CCND1 rearrangement. This high concordance between karyotype and FISH suggests that karyotype is reliable in identifying 11q13 translocations. Loss of $Y$ chromosome was confirmed by FISH in all six cases and loss of $X$ chromosome in a single case (Table 1). All five cases with loss of chromosome 1 were confirmed by FISH. Four cases that were karyotypic negative for loss of $\mathrm{Y}$ was also negative by FISH. However, FISH analysis identified 


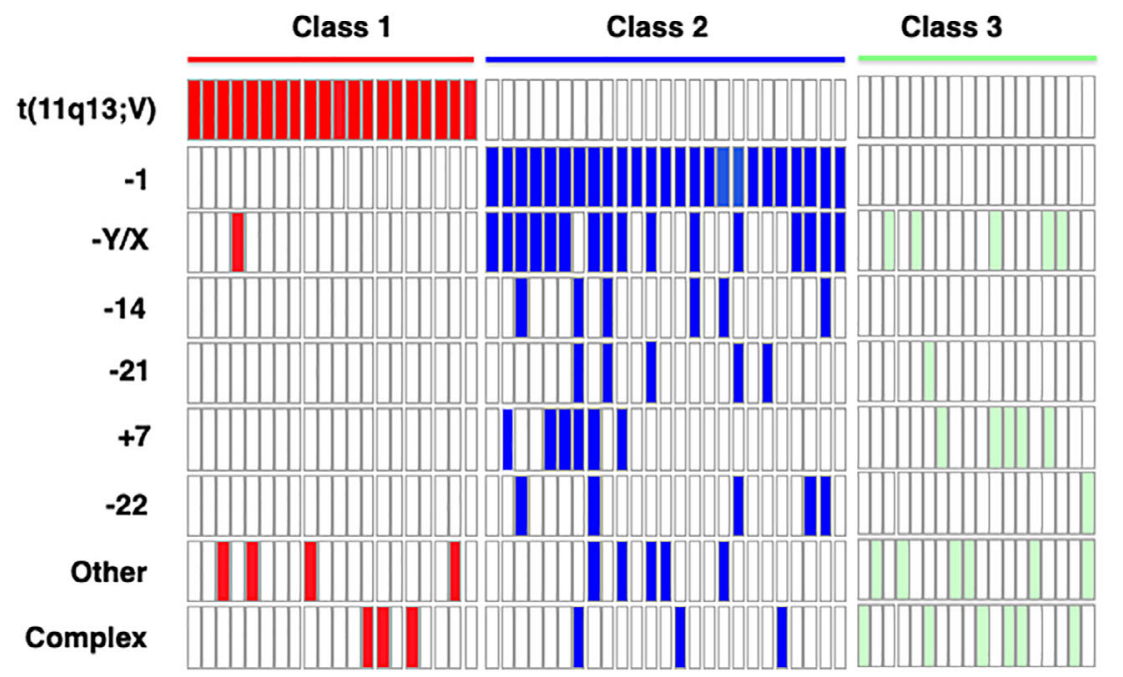

FIGURE 1 Patterns of chromosome abnormalities in various classes of renal oncocytomas. Red, class 1; blue, class 2; light green, class 3. Light blue in class 2 indicates partial deletion of chromosome arm 1p
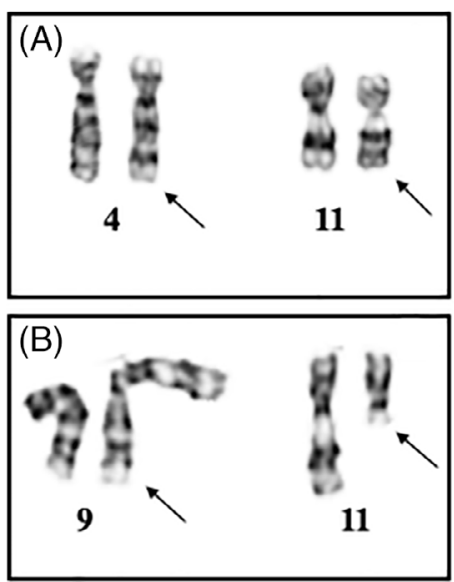
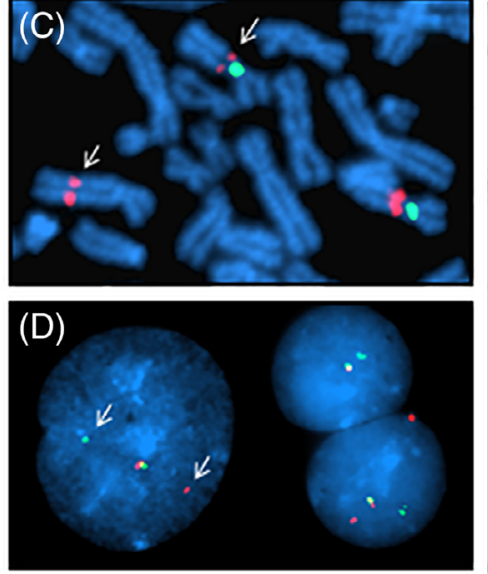

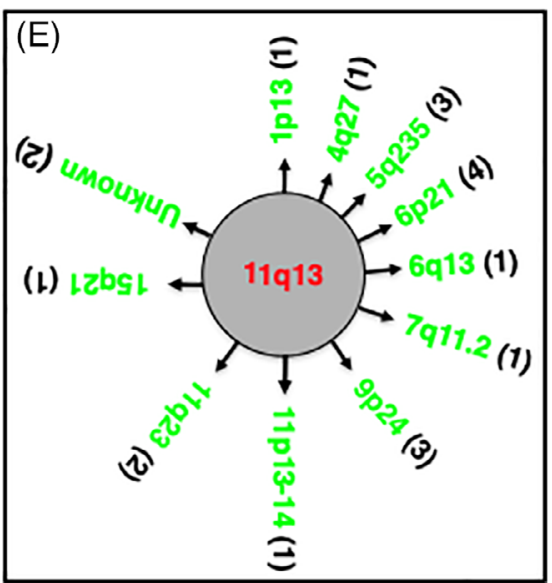

FIGURE 2 Cytogenetic and FISH analyses of class 1 RO. Partial karyotypes showing 11q13 rearrangements with (A) 4q and (B) 9p (arrows indicate rearranged chromosomes). FISH analysis using the CCND1 (orange)/CEP 11 (green) probe (C, partial metaphase) and the two-color CCND1 break apart probe (D, interphase). Rearranged CCND1 is shown by white arrows. E, The frequency of 11q13 translocation rearrangement partners

monosomy 1 in $84 \%$ cells and loss of $Y$ in $3 \%$ cells in a case that showed only loss of $Y$ by karyotype (case no. 10-048, Table S1), suggesting that the loss of chromosome 1 clone could not be identified by karyotype analysis due likely only normal cells grew. Loss $Y$ in this case may be age-related chromosome alteration.

To test further the possibility of using FISH alone as a diagnostic test, we have examined randomly selected karyotypically normal and failure RO cases by FISH using probes for chromosomes X, Y, 1, and CCND1. We identified 6 of 22 (27\%) with CCND1 rearrangement, 6 of $15(40 \%)$ with loss of chromosome 1, 5 of 18 (28\%) cases with loss of $\mathrm{Y}$. These data therefore suggest that FISH using a panel of probes targeting the most recurrent chromosome abnormalities may serve as diagnostic test for identifying classes 1 and 2 ROs.

\section{3 | Clinical correlations}

No clinical and histological correlations were identified between various classes of ROs, except age and WGD. Ages between the RO classes were statistically significant, where class 1 ROs occur in younger patients (class 1, 55.07 years; class 2, 64.6 years; class $3,69.5$ years) $(P=.0014)$. Frequencies of WGD between RO classes are also statistically significant with class 2 exhibiting a higher frequency $(P=.01)$.

\section{4 | Validation of the present data with Mitelman database}

A total of 102 karyotypically abnormal ROs were reported in Mitelman database. To identify the type and frequency of chromosome abnormalities characteristic of each class of RO, we chose 91 cases after excluding 11 cases reported by us before. We compared frequencies of chromosome abnormalities with our series of 63 cases (Tables 1 and 2). Overall this analysis showed similar types and frequencies of chromosome aberrations in all three classes of ROs. Interestingly, partial deletion of chromosome arm 1p in class $1 \mathrm{RO}$ was present in two tumors in the present series and in one case 
in the Mitelman database. WGD in class 2 RO was found in eight cases in our series and five in the Mitelman database.

This analysis further confirms that $11 q 13$ rearrangements occur as a sole abnormality in the majority of the class $1 \mathrm{RO}$. The chromosome 1 and sex chromosome losses occur together in most cases of class 2 RO. Loss of $Y$ was seen as a sole abnormality in class $3 R O$ in 4 of $16(25 \%)$ in our series and 5 of $51(10 \%)$ in the Mitelman database. Loss of $Y$ can be an age-associated abnormality. Trisomy 7 was found to be a sole abnormality in class $3 \mathrm{RO}$ in three of 16 (19\%) in our series and 5 of 51 (10\%) in the Mitelman database. Trisomy 7 is a nonspecific change that can be seen in proliferative kidney epithelial cells. Among the structural abnormalities, $\mathrm{t}(6 ; 9)(\mathrm{p} 12 ; \mathrm{p} 23-24)$ and $19 \mathrm{p} 13$ rearrangement was seen in four cases each of the class $3 \mathrm{RO}$ in the Mitelman database and one each in our series.

\section{4 | DISCUSSION}

Karyotypic characterization of chromosome aberrations has greatly contributed in our understanding of the biological events underlying the origin and progression of major histologic subtypes clear cell and papillary renal cell cancer. ${ }^{6-11}$ Various recurrent nonrandom chromosome changes reflecting copy number alterations and mutations have been identified in clear cell, papillary, and chromophobe RCCs. ${ }^{12-14}$ However, the genetic and molecular basis of sporadic RO is relatively poorly understood. Only a small number of cases with karyotypic aberrations have been reported in the literature.,15-18 These and other molecular studies have identified two major groups of RO, one with monosomy 1 accompanied by losses of $Y$ in males or $\mathrm{X}$ chromosome in females, and a second one with 11q13 rearrangement. ${ }^{19}$ Here we report the largest series of karyotypically characterized RO that revealed three distinctly different and mutually exclusive classes of chromosome abnormalities. We have previously reported a small series of RO cases where we showed 11q13 rearrangements and loss of chromosome 1 as mutually exclusive cytogenetic aberrations. ${ }^{5}$

The rearrangement of 11q13 with multiple translocation partners in the genome is a distinct feature of class $1 \mathrm{RO}$. Others and we have previously shown that CCND1 at 11q13 is the target of rearrangement with the breakpoints outside of the coding region resulting in increased expression of cyclin D1.,18-20 CCND1 chromosomal rearrangement and amplification results in over expression of cyclin D1 in many tumor types. ${ }^{21}$ CCND1 translocation due to juxtaposition to immunoglobulin genes acts as a driver mutation in several forms of malignant lymphomas. ${ }^{22,23}$ CCND1 also participates in translocations with other genes than immunoglobulin genes in other tumors resulting in alternative mechanisms of cyclin D1 overexpression. ${ }^{24,25}$ However, the gene(s) or genomic sequences that drive the deregulated expression of cyclin D1 in RO are not known. As 11q13 rearrangements occur with multiple chromosomal partners in class $1 \mathrm{RO}$, it is likely that enhancers similar to immunoglobulin-type genes may be involved in these translocations. The transcriptional activation mechanisms of CCND1 deregulation in RO remains to be examined. In hematologic malignancies, there are number of genes (eg, KMT2A and BCL6) known to be involved in translocations with multiple partners in the genome. As CCND1/11q13 translocations with multiple genomic regions occur as a sole abnormality in RO, they are likely to act as driver mutations.

Loss of chromosome 1 in association with sex chromosome loss is characteristic feature of class $2 \mathrm{RO}$. This class of RO shows a male predominance (68\% males vs $32 \%$ females) with more frequent loss of $Y$ in males (71\% cases) compared to loss of $X$ in females (38\%). The biological consequence of chromosome 1 loss is not well understood. However, no significant differences in expression of genes located on chromosome 1 have been found in a small series of RO cases reported recently. ${ }^{19}$ Interestingly, we found two cases showing a common deletion at 1p22-p34 (one with deletion distal to 1p21 and other with an interstitial deletion at 1p22-p34). This limited number of cases with deletion at 1p22-p34 potentially points to a critical region of deletion that might harbor important genes. However, larger series of class $2 \mathrm{RO}$ cases need to be studied in order to identify if a critical region of deletion on chromosome 1 exists. The biological consequence of chromosome losses of $\mathrm{Y}$ or $\mathrm{X}, 14,21$, and 22 also remains unknown. Although it is well established that abnormalities involving chromosome 1 are frequent in a wide-variety of tumors, the biologic consequences of these karyotypic aberrations remain poorly understood.

Class 3 RO comprising 18 tumors showed simple ( $\leq 3$ changes) or complex karyotypes ( $\geq 4$ changes) with gains, losses, and structural rearrangements. No class 3 tumors had 11q13 translocations or chromosome 1 abnormalities. Thirteen tumors showed simple karyotypes and five had complex karyotypes. The only recurrent changes identified were loss of $Y$ or $X$ in seven cases, gain of chromosome 7 in five cases, and structural rearrangements at $2 q 37$ in three cases. Loss of the $Y$ chromosome and/or trisomy 7 was seen in seven cases. These abnormalities are nonspecific and can occur in benign proliferative epithelial cells. Interestingly, telomeric associations (tas) were seen in three tumors. It is unclear what the significance of these seemingly nonrecurrent chromosome abnormalities is in class 3 RO. Subjecting these tumors to extensive genomic characterization may shed some light on the genetic basis of this class of RO.

In the present series of RO, we observed WGD in both classes 1 and 2 , but not in class 3 tumors. It was most frequent in class 2 with $32 \%$ tumors exhibiting WGD. Tetraploidy resulting from genome doubling is a common phenomenon in many cancer types. It has been hypothesized that tetraploidization in cancer is an intermediate step before aneuploidy onset, early event in tumor progression, and occurs in transition from premalignant state to malignant state. ${ }^{14,26,27}$ In 9 of 10 RO tumors with evidence for WGD it was only present in subclones, suggesting that duplication occurred as a later event. Consistent with this, tetraploid clones have been reported to occur later in tumor development, ${ }^{28}$ as well as both before and after other copy number alterations in different tumor types. ${ }^{29}$ It has also been shown that tetraploid cells exhibit tolerance of chromosomal instability compared to diploid clones and associate with poor prognosis in colorectal tumors. ${ }^{26}$ As ROs are benign, whether the presence of WGD fuels further genomic changes accelerating tumor progression is unknown. 
We observed tas involving various chromosomes in four cases (three class 3 and one class 1 tumors) of ROs. Such telomeric associations have also been identified in three previously published cases of class 3 RO. ${ }^{30,31}$ Telomeric associations caused by end-to-end fusions of chromosome ends have been shown to be frequently associated with cancer. ${ }^{32,33}$ Defects in telomere function was identified as the cause for the formation of dicentric chromosomes created through end-to-end fusions, ${ }^{34}$ specifically mediated by telomeric DNA-binding protein, TRF2. ${ }^{35}$ However, the causes and consequences of telomeric associations seen in the present study of RO remain to be explored.

In the present study, we performed extensive karyotypic and FISH analyses of large number of human ROs. These analyses identified three karyotypically distinct classes of oncocytomas. Overall, our data emphasize the importance of cytogenetic analysis in characterization and differential diagnosis of oncocytomas from chromophobe renal cancer. The identification of distinct karyotypic classes of RO can provide a basis in further characterization of these tumors in exploring potential mechanisms of oncogenesis in these tumors.

\section{ORCID}

Vundavalli V. Murty (D) https://orcid.org/0000-0003-1603-6414

\section{REFERENCES}

1. Perez-Ordonez B, Hamed G, Campbell S, et al. Renal oncocytoma: a clinicopathologic study of 70 cases. Am J Surg Pathol. 1997;21: 871-883.

2. Nickerson ML, Warren MB, Toro JR, et al. Mutations in a novel gene lead to kidney tumors, lung wall defects, and benign tumors of the hair follicle in patients with the Birt-Hogg-Dube syndrome. Cancer Cell. 2002;2:157-164.

3. van den Berg E, van der Hout AH, Oosterhuis JW, et al. Cytogenetic analysis of epithelial renal-cell tumors: relationship with a new histopathological classification. Int J Cancer. 1993;55:223-227.

4. Mitelman F, Johansson B, Mertens F. (2017). Mitelman Database of Chromosome Aberrations and Gene Fusions in Cancer. http://cgap. nci.nih.gov/Chromosomes/Mitelman.

5. Jhang JS, Narayan G, Murty VV, Mansukhani MM. Renal oncocytomas with 11q13 rearrangements: cytogenetic, molecular, and immunohistochemical analysis of cyclin D1. Cancer Genet Cytogenet. 2004;149:114-119.

6. Antonelli A, Tardanico R, Balzarini P, et al. Cytogenetic features, clinical significance and prognostic impact of type 1 and type 2 papillary renal cell carcinoma. Cancer Genet Cytogenet. 2010;199:128-133.

7. Carroll PR, Murty VV, Reuter V, et al. Abnormalities at chromosome region 3p12-14 characterize clear cell renal carcinoma. Cancer Genet Cytogenet. 1987;26:253-259.

8. de Oliveira D, Dall'Oglio MF, Reis ST, et al. Chromosome 9p deletions are an independent predictor of tumor progression following nephrectomy in patients with localized clear cell renal cell carcinoma. Urol Oncol. 2014;32:601-606.

9. Klatte T, Rao PN, de Martino M, et al. Cytogenetic profile predicts prognosis of patients with clear cell renal cell carcinoma. J Clin Oncol. 2009;27:746-753.

10. Presti JC Jr, Rao PH, Chen Q, et al. Histopathological, cytogenetic, and molecular characterization of renal cortical tumors. Cancer Res. 1991;51:1544-1552.
11. Szponar A, Zubakov D, Pawlak J, Jauch A, Kovacs G. Three genetic developmental stages of papillary renal cell tumors: duplication of chromosome 1q marks fatal progression. Int J Cancer. 2009;124: 2071-2076.

12. Cancer Genome Atlas Research Network, Linehan WM, Spellman PT, et al. Comprehensive molecular characterization of papillary renal-cell carcinoma. N Engl J Med. 2016;374:135-145.

13. Creighton $\mathrm{CJ}$, Morgan $\mathrm{M}$, Gunaratne $\mathrm{PH}$, et al. Comprehensive molecular characterization of clear cell renal cell carcinoma. Nature. 2013;499: 43-49.

14. Durinck S, Stawiski EW, Pavia-Jimenez A, et al. Spectrum of diverse genomic alterations define non-clear cell renal carcinoma subtypes. Nat Genet. 2015;47:13-21.

15. Dijkhuizen $\mathrm{T}$, van den Berg E, Storkel S, et al. Renal oncocytoma with $\mathrm{t}(5 ; 12 ; 11)$, der(1)(1;8) and add(19): "true" oncocytoma or chromophobe adenoma? Int J Cancer. 1997;73:521-524.

16. Mertz KD, Demichelis F, Sboner A, et al. Association of cytokeratin 7 and 19 expression with genomic stability and favorable prognosis in clear cell renal cell cancer. Int J Cancer. 2008;123:569-576.

17. Paner GP, Lindgren V, Jacobson K, Harrison K, et al. High incidence of chromosome 1 abnormalities in a series of 27 renal oncocytomas: cytogenetic and fluorescence in situ hybridization studies. Arch Pathol Lab Med. 2007;131:81-85.

18. Sukov WR, Ketterling RP, Lager DJ, et al. CCND1 rearrangements and cyclin D1 overexpression in renal oncocytomas: frequency, clinicopathologic features, and utility in differentiation from chromophobe renal cell carcinoma. Hum Pathol. 2009;40:1296-1303.

19. Joshi S, Tolkunov $\mathrm{D}$, Aviv $\mathrm{H}$, et al. The genomic landscape of renal Oncocytoma identifies a metabolic barrier to tumorigenesis. Cell Rep. 2015;13:1895-1908

20. Gopal RK, Calvo SE, Shih AR, et al. Early loss of mitochondrial complex $I$ and rewiring of glutathione metabolism in renal oncocytoma. Proc Natl Acad Sci USA. 2018;115(27):E6283-E6290.

21. Zukerberg LR, Yang WI, Gadd M, et al. Cyclin D1 (PRAD1) protein expression in breast cancer: approximately one-third of infiltrating mammary carcinomas show overexpression of the cyclin D1 oncogene. Mod Pathol. 1995;8:560-567.

22. Callanan M, Leroux D, Magaud JP, Rimokh R. Implication of cyclin D1 in malignant lymphoma. Crit Rev Oncog. 1996;7:191-203.

23. Rocha CK, Praulich I, Gehrke I, Hallek M, Kreuzer KA. A rare case of $t(11 ; 22)$ in a mantle cell lymphoma like B-cell neoplasia resulting in a fusion of IGL and CCND1: case report. Mol Cytogenet. 2011;4:8.

24. Masamha CP, Albrecht TR, Wagner EJ. Discovery and characterization of a novel CCND1/MRCK gene fusion in mantle cell lymphoma. J Hematol Oncol. 2016;9:30.

25. Menke JR, Vasmatzis G, Murphy S, et al. Mantle cell lymphoma with a novel $t(11 ; 12)(q 13 ; p 11.2)$ : a proposed alternative mechanism of CCND1 upregulation. Hum Pathol. 2017;64:207-212.

26. Dewhurst SM, McGranahan N, Burrell RA, et al. Tolerance of wholegenome doubling propagates chromosomal instability and accelerates cancer genome evolution. Cancer Discov. 2014;4:175-185.

27. Olaharski AJ, Sotelo R, Solorza-Luna G, et al. Tetraploidy and chromosomal instability are early events during cervical carcinogenesis. Carcinogenesis. 2006;27:337-343.

28. Gerlinger M, Rowan AJ, Horswell S, et al. Intratumor heterogeneity and branched evolution revealed by multiregion sequencing. $N$ Engl $J$ Med. 2012;366:883-892

29. Zack TI, Schumacher SE, Carter SL, et al. Pan-cancer patterns of somatic copy number alteration. Nat Genet. 2013;45:1134-1140.

30. Kovacs G, Muller-Brechlin R, Szucs S. Telomeric association in two human renal tumors. Cancer Genet Cytogenet. 1987;28: 363-366.

31. van den Berg E, Dijkhuizen T, Storkel S, et al. Chromosomal changes in renal oncocytomas. Evidence that $t(5 ; 11)(q 35 ; q 13)$ may characterize 
a second subgroup of oncocytomas. Cancer Genet Cytogenet. 1995;79: 164-168.

32. Lin TT, Letsolo BT, Jones RE, Rowson J, et al. Telomere dysfunction and fusion during the progression of chronic lymphocytic leukemia: evidence for a telomere crisis. Blood. 2010;116:1899-1907.

33. Tanaka $\mathrm{H}$, Abe $\mathrm{S}$, Huda $\mathrm{N}$, et al. Telomere fusions in early human breast carcinoma. Proc Natl Acad Sci U S A. 2012;109:1409814103.

34. Counter CM, Avilion AA, LeFeuvre CE, et al. Telomere shortening associated with chromosome instability is arrested in immortal cells which express telomerase activity. EMBO J. 1992;11: 1921-1929.

35. van Steensel B, Smogorzewska A, de Lange T. TRF2 protects human telomeres from end-to-end fusions. Cell. 1998;92:401-413.

\section{SUPPORTING INFORMATION}

Additional supporting information may be found online in the Supporting Information section at the end of this article.

How to cite this article: Anderson CB, Lipsky M, Nandula SV, et al. Cytogenetic analysis of 130 renal oncocytomas identify three distinct and mutually exclusive diagnostic classes of chromosome aberrations. Genes Chromosomes Cancer. 2019;

1-7. https://doi.org/10.1002/gcc.22766 